\title{
Approximate Solution of Nonlinear Klein-Gordon Equation Using Sobolev Gradients
}

\author{
Nauman Raza, ${ }^{1}$ Asma Rashid Butt, ${ }^{2}$ and Ahmad Javid ${ }^{1}$ \\ ${ }^{1}$ Department of Mathematics, University of the Punjab, Lahore 54590, Pakistan \\ ${ }^{2}$ Department of Mathematics, University of Engineering and Technology, Lahore 54890, Pakistan
}

Correspondence should be addressed to Asma Rashid Butt; asma.rbutt@gmail.com

Received 4 November 2015; Revised 1 February 2016; Accepted 2 February 2016

Academic Editor: Mark A. McKibben

Copyright (c) 2016 Nauman Raza et al. This is an open access article distributed under the Creative Commons Attribution License, which permits unrestricted use, distribution, and reproduction in any medium, provided the original work is properly cited.

\begin{abstract}
The nonlinear Klein-Gordon equation (KGE) models many nonlinear phenomena. In this paper, we propose a scheme for numerical approximation of solutions of the one-dimensional nonlinear KGE. A common approach to find a solution of a nonlinear system is to first linearize the equations by successive substitution or the Newton iteration method and then solve a linear least squares problem. Here, we show that it can be advantageous to form a sum of squared residuals of the nonlinear problem and then find a zero of the gradient. Our scheme is based on the Sobolev gradient method for solving a nonlinear least square problem directly. The numerical results are compared with Lattice Boltzmann Method (LBM). The $L_{2}, L_{\infty}$, and Root-Mean-Square (RMS) values indicate better accuracy of the proposed method with less computational effort.
\end{abstract}

\section{Introduction}

Many nonlinear phenomena in solid state physics, plasma physics, electrostatics, chemical kinetics, and fluid dynamics can be modeled through partial differential equations (PDEs) [1]. One example of such a PDE is the nonlinear KGE which arises in relativistic quantum mechanics and field theory [2]. This equation has attracted the attention of many scientists and has been widely used to study various laws related to motions of elementary particles in condensed matter and particle physics [3]. The KGE models a wide class of scientific phenomena, including propagation of dislocations in crystals and the behavior of elementary particles. The equation also arises in the study of solitons and perturbation theory [4-6].

The initial value problem for the one-dimensional nonlinear KGE is given by

$$
u_{t t}+\alpha u_{x x}+g(u)=f(x, t)
$$

where $u=u(x, t)$ shows the wave displacement at position $x$ at time $t, \alpha$ is constant, and $g(u)$ is nonlinear force. The residual of the equation can be written as

$$
M(u)=u_{t t}+\alpha u_{x x}+g(u)-f(x, t) .
$$

The nonlinear KGE also has a conserved Hamiltonian energy:

$$
E(u)=\int\left(\frac{1}{2} u_{t}^{2}+\frac{1}{2} u_{x}^{2}+G(u)\right),
$$

where $G^{\prime}(u)=g(u)$.

In this paper we find the numerical approximation of the nonlinear KGE with quadratic nonlinearity

$$
u_{t t}+\alpha u_{x x}+\beta u+\gamma u^{2}=f(x, t),
$$

and also with cubic nonlinearity

$$
u_{t t}+\alpha u_{x x}+\beta u+\gamma u^{3}=f(x, t),
$$

where $\alpha, \beta$, and $\gamma$ are known constants.

A number of different numerical schemes have been proposed for the solution of the KGE. It is mentioned in [1] that many methods like inverse scattering method, Bäcklund transformation, the auxiliary equation method [7, 8], pseudospectral method, and Riccati equation expansion methods have been used to solve these types of equations (see [1] and references therein). Other methods like spectral and pseudospectral approaches have recently been presented in [9]. In [10] Spectral methods using Legendre polynomials 
(SMLP) as basis functions are considered to solve the KGE. The solution of the nonlinear KGE, using $\theta$-weighted finite difference discretization with radial basis functions (RBS) is discussed in [11]. Many difference schemes have been given in [12]. In these difference schemes, undesirable results like instability and loss of spatial symmetry for large number of parameters in initial conditions were observed.

Another approach to solving any boundary value problem is to formulate the problem in terms of minimizing a least square functional representing the residuals of the equation. The least square functional acts as an error representative. The boundary conditions are conveniently satisfied in this gradient descent approach.

One such technique is the Sobolev gradient method. Sobolev gradients have been used in minimization of Ginzburg-Landau energy functionals [13] related to phase separation and ordering in finite difference settings [14], electrostatic potential equations [15], nonlinear elliptic problems [16], inverse problems in elasticity [17], groundwater modeling [18], and simulation of Bose Einstein condensates [19].

The paper is organized as follows. In Section 2 Sobolev gradient method is discussed and some existence results for the proposed method are presented. In Section 3 we apply the method on the nonlinear KGE. In Section 4 results of the numerical experiments are presented by solving some numerical examples. Comparison with other standard methods like spectral or finite difference method is also given. Section 5 contains the summary and conclusion of the results obtained. And at the end some references are provided.

In this paper all the numerical experiments are carried out using Intel(R) Core i3 $1.70 \mathrm{GHz}$ processor with $4 \mathrm{~GB}$ RAM. All the codes and graphs are drawn in MATLAB and are available upon request.

\section{Sobolev Gradient Method}

In this section we throw some light on Sobolev gradients and steepest descent. A comprehensive procedure for the interpretation of Sobolev gradients is presented in [13] and the given theory is borrowed from [13].

Here we are giving the Riesz Representation theorem which is useful in our work.

Theorem 1. Every bounded linear functional $F$ on a Hilbert space $H$ can be represented in terms of the inner product; namely,

$$
F(x)=\langle x, z\rangle,
$$

where $z$ depends on $F$, is uniquely determined, and has norm $\|z\|=\|F\|$.

Let $m$ be a positive integer and $F$ be a real-valued $C^{(1)}$ function on $R^{m}$. Then the gradient $\nabla F$ is defined as

$$
\begin{gathered}
\lim _{t \rightarrow 0} \frac{1}{t}(F(x+t h)-F(x))=F^{\prime}(x) h \\
=\langle h,(\nabla F)(x)\rangle_{R^{m}}, \quad x, h \in R^{m} .
\end{gathered}
$$

For $F$ as mentioned above but with an inner product $\langle\cdot, \cdot\rangle_{s}$ different from the standard inner product $\langle\cdot, \cdot\rangle_{R^{m}}$, there is a function

$$
\nabla_{s} F: R^{m} \longrightarrow R^{m}
$$

so that

$$
F^{\prime}(x) h=\left\langle h,\left(\nabla_{s} F\right)(x)\right\rangle_{s}, \quad x, h \in R^{m} .
$$

Since every linear functional defined on finite-dimensional vector space is bounded so by Theorem 1 the linear functional $F^{\prime}(x)$ can be represented using any inner product on $R^{m}$. Saying that $\nabla_{s} F$ is the gradient of $F$ with respect to the inner product $\langle\cdot, \cdot\rangle_{s}$ it is worth noticing that the gradient $\nabla_{s} F(x)$ has the same set of properties as that of $\nabla F$.

From linear algebra there is a linear transformation

$$
T: R^{m} \longrightarrow R^{m}
$$

by which these two inner products are related by

$$
\langle x, y\rangle_{s}=\langle x, T y\rangle_{R^{m}} ; \quad x, y \in R^{m}
$$

and some reflection leads to

$$
\left(\nabla_{s} F\right)(x)=T^{-1}(\nabla F)(x), \quad x \in R^{m} .
$$

Every point $x \in R^{m}$ possesses its own inner product $\langle\cdot, \cdot\rangle_{x}$ on $R^{m}$. Therefore, for $x \in R^{m}$, define

$$
\nabla_{x} F: R^{m} \longrightarrow R^{m}
$$

such that

$$
F^{\prime}(x) h=\left\langle h,\left(\nabla_{x} F\right)(x)\right\rangle_{x}, \quad x, h \in R^{m} .
$$

We have a collection of gradients which have tremendous differences in numerical properties for a function $F$ depending upon the choice of metric.

The gradient of a function $F$ defined in a finite or infinite dimensional Sobolev space is known as a Sobolev gradient.

The method of steepest descent can be classified into two categories: continuous steepest descent and discrete steepest descent.

Consider an inner product $\langle\cdot, \cdot\rangle_{s}$, a real-valued $C^{(1)}$ function $F$, and its gradient $\nabla_{s} F$ defined on a Hilbert space $H$.

Discrete steepest descent generates a sequence of points $x_{m}$ such that

$$
x_{m}=x_{m-1}-\delta_{m-1}\left(\nabla_{s} F\right)\left(x_{m-1}\right), \quad m=1,2, \ldots,
$$

where initial guess $x_{0}$ is given and $\delta_{m-1}$ is selected so that it minimizes, if possible,

$$
F\left(x_{m-1}-\delta\left(\nabla_{s} F\right)\left(x_{m-1}\right)\right), \quad \delta \in R .
$$

Continuous steepest descent is a process of finding a function $z:[0, \infty) \rightarrow H$ such that

$$
\frac{d z}{d t}=-\nabla F(z(t)), \quad z(0)=z_{\text {initial }}
$$


Continuous steepest descent is regarded as a limiting case of discrete steepest descent and (15) can be used as a numerical technique to extract solutions of (17).

To prove the convergence of discrete steepest descent, we extract a theoretical initial point by using continuous steepest descent.

By (15) we desire a $u=\lim _{k \rightarrow \infty} x_{k}$, so that

$$
\begin{aligned}
F(u) & =0 \\
\text { or } \nabla_{s} F(u) & =0 .
\end{aligned}
$$

By (17), we desire a $u=\lim _{t \rightarrow \infty} z(t)$ so that (18) is satisfied.

The Sobolev gradient technique is a process in which we discretize a gradient in a Sobolev space rather than in a Euclidean space. For the solution of a PDE, we represent PDE using an error functional $F$ for a least square formulation. We try to find a $u$ in the domain of the PDE such that residual $M$ is zero, while $F$ and its gradient are given by

$$
\begin{aligned}
F & =\frac{1}{2}\|M\|^{2}, \\
\nabla F & =M^{\prime}(u) M(u) .
\end{aligned}
$$

The following theorem gives the existence and convergence of several linear and nonlinear forms of $F$.

Theorem 2. Let $F$ be a nonnegative $C^{(1)}$ function (a differentiable function whose derivative is continuous) on a Hilbert space $H$ which has a locally Lipschitzian gradient. Then, for each $x \in H$, there exists a unique function $z:[0, \infty) \rightarrow H$ with

$$
\begin{aligned}
& z(0)=x \\
& z^{\prime}(t)=-\left(\nabla_{s} F\right) z(t), \quad t \geq 0 .
\end{aligned}
$$

Another observation which is useful in our work is as follows.

Theorem 3. Suppose all the conditions in the hypothesis of the above theorem are satisfied and

$$
\lim _{t \rightarrow \infty} z(t)=u
$$

exists; then

$$
\nabla_{s} F(z(t))=0
$$

\section{The Nonlinear Klein-Gordon Equation}

Consider the problem

$$
\begin{aligned}
\frac{\partial^{2} u}{\partial t^{2}}+\alpha \frac{\partial^{2} u}{\partial x^{2}}+\beta u+\gamma u^{k}= & f(x, t), \\
& x \in \Omega=[a, b], 0<t \leq T
\end{aligned}
$$

with initial conditions

$$
\begin{gathered}
u(x, 0)=h_{1}(x), \quad x \in \Omega \\
u_{t}(x, 0)=h_{2}(x), \quad x \in \Omega,
\end{gathered}
$$

and Dirichlet boundary conditions

$$
u(x, t)=g(x, t), \quad x \in \partial \Omega, 0<t \leq T,
$$

where $\alpha, \beta$, and $\gamma$ are constants and $f, h_{1}, h_{2}$, and $g$ are known functions while $u$ is unknown. For $k=2,3$ we have quadratic and cubic nonlinearity, respectively.

To solve the problem numerically, we work in a finitedimensional vector space $R^{N}$ on a uniform grid. We denote by $L_{2}$ or $H_{0}^{2}$ the vector space $R^{N}$ equipped with the usual inner product $\langle x, y\rangle=\sum_{i} x(i) y(i)$. The operators $D_{00}, D_{11}, D_{22}$ : $R^{N} \rightarrow R^{N-2}$ are defined by

$$
\begin{aligned}
& D_{00}(u)(i)=\frac{(u(i+2)+2 u(i+1)+u(i))}{4}, \\
& D_{11}(u)(i)=\frac{u(i+2)-u(i)}{2 \delta_{x}}, \\
& D_{22}(u)(i)=\frac{(u(i+2)-2 u(i+1)+u(i))}{\delta_{x}^{2}}
\end{aligned}
$$

for $i=1,2, \ldots, N-2$ and where $\delta_{x}=b-a /(N-1)$ is the spacing between the nodes. $D_{00}$ is the average operator. $D_{11}$ and $D_{22}$ are standard central difference formulas for approximating the first and second derivatives. The choice of difference formula is not important to the theoretical development of this paper; other choices would also work.

The numerical version of the problem of evolving from a time $t$ to $t+\delta_{t}$ is to solve

$$
\begin{aligned}
& D_{00}\left(\left(1+\beta \delta_{t}^{2}\right) u-2 f_{1}+f_{2}+\gamma \delta_{t}^{2} u^{k}\right)+\alpha \delta_{t}^{2} D_{22} u \\
& \quad=0
\end{aligned}
$$

where $f_{1}$ and $f_{2}$ in the equation are $u$ at the previous and present time steps and $u$ is the $u$ desired at the next time. We can put the solution of this problem in terms of minimizing a functional via steepest descent. Define $M \in R^{N-2}$ by

$$
\begin{aligned}
M= & D_{00}\left(\left(1+\beta \delta_{t}^{2}\right) u-2 f_{1}+f_{2}+\gamma \delta_{t}^{2} u^{k}\right) \\
& +\alpha \delta_{t}^{2} D_{22} u,
\end{aligned}
$$

where $M$ represents the residual of the equation and when it approaches zero we have the desired $u$. The least square functional associated with the residual is given by

$$
F(u)=\frac{1}{2}\langle M(u), M(u)\rangle .
$$

$F$ has a minimum of nearly zero when $M(u)$ approaches zero. So we will look for the minimum of this functional.

3.1. Gradients and Minimization. The gradient $\nabla F(u) \in R^{N}$ of a functional $F(u)$ in $L_{2}$ is found by solving

$$
F(u+h)=F(u)+\langle\nabla F(u), h\rangle+O\left(h^{2}\right)
$$

for test functions $h$. The gradient points in the direction of greatest increase of the functional. The direction of greatest 
TABle 1: Comparison of solution for Example 4 using Euclidean and Sobolev Gradients with $\delta_{t}=0.2, t=1$, and $c=0.05$.

\begin{tabular}{|c|c|c|c|c|c|c|}
\hline \multirow{2}{*}{$N$} & \multicolumn{3}{|c|}{$L_{2}$ gradient } & \multicolumn{3}{|c|}{$H_{2}^{2}$ gradient } \\
\hline & $\lambda$ & Iterations & CPUs & $\lambda$ & Iterations & CPUs \\
\hline 10 & $1.0 \times 10^{-4}$ & 36015 & 0.1228 & 1.0 & 6469 & 1.4608 \\
\hline 20 & $1.0 \times 10^{-5}$ & 228336 & 6.6846 & 1.0 & 8060 & 4.7661 \\
\hline 50 & $1.0 \times 10^{-6}$ & 2181749 & 99.6085 & 1.0 & 9985 & 26.0143 \\
\hline 70 & - & - & - & 1.0 & 10671 & 63.8609 \\
\hline
\end{tabular}

TABLE 2: Comparison of $L_{\infty}, L_{2}$, and RMS errors for Example 4 at $c=0.5$ between Sobolev gradient method and LBM [20].

\begin{tabular}{|c|c|c|c|c|c|c|c|c|c|c|}
\hline \multirow{2}{*}{$t$} & \multicolumn{5}{|c|}{ Sobolev descent } & \multicolumn{5}{|c|}{ LBM } \\
\hline & Nodes & $\delta_{t}$ & $L_{\infty}$ & $L_{2}$ & RMS & Nodes & $\delta_{t}$ & $L_{\infty}$ & $L_{2}$ & RMS \\
\hline 1 & 20 & 0.1 & $3.1518 \times 10^{-4}$ & $9.3185 \times 10^{-4}$ & $2.0838 \times 10^{-4}$ & 100 & $5.0 \times 10^{-5}$ & $1.4189 \times 10^{-4}$ & $6.6508 \times 10^{-4}$ & $6.6171 \times 10^{-5}$ \\
\hline 2 & 10 & 0.2 & $2.4966 \times 10^{-4}$ & $5.4618 \times 10^{-4}$ & $1.7272 \times 10^{-4}$ & 100 & $5.0 \times 10^{-5}$ & $4.6601 \times 10^{-4}$ & $1.5438 \times 10^{-3}$ & $1.5362 \times 10^{-4}$ \\
\hline 3 & 10 & 0.2 & $8.5185 \times 10^{-4}$ & $1.8000 \times 10^{-3}$ & $5.6788 \times 10^{-4}$ & 100 & $5.0 \times 10^{-5}$ & $1.9445 \times 10^{-3}$ & $4.9588 \times 10^{-3}$ & $4.9342 \times 10^{-4}$ \\
\hline 4 & 20 & 0.1 & $3.400 \times 10^{-3}$ & $1.0300 \times 10^{-2}$ & $2.3000 \times 10^{-3}$ & 100 & $5.0 \times 10^{-5}$ & $2.8219 \times 10^{-2}$ & $7.1870 \times 10^{-2}$ & $7.1513 \times 10^{-3}$ \\
\hline
\end{tabular}

decrease of the functional is $-\nabla F(u)$. This is the corner stone of steepest descent algorithms.

We replace $u$ by $u-\lambda \nabla F$ to reduce $F(u)$, where $\lambda$ is a positive number. This process is repeated until $F(u)$ or $\nabla F$ are reduced by a fair margin. To satisfy the boundary conditions in the finite-dimensional analogue of the original problem we use a projection $\phi: R^{N} \rightarrow R^{N}$ which projects vectors in $R^{N}$ onto the subspace in which the initial and final entries of vectors are zero. We will use $\phi \nabla F(u)$ instead of using $\nabla F(u)$. In this particular case,

$$
\begin{aligned}
\phi \nabla F(u)= & \phi\left(1+\beta \delta_{t}^{2}+k \gamma \delta_{t}^{2} u^{k-1}\right) D_{00}^{t}(M(u)) \\
& +\alpha \delta_{t}^{2} D_{22}^{t}(M(u))
\end{aligned}
$$

gives the desired gradient in $L_{2}$.

For a nonlinear problem like the KGE the $L_{2}$ gradient or Euclidean gradient is not a good choice. Standard steepest descent using the $L_{2}$ gradient is inefficient when the spacing of the grid is refined and also when the dimensions of the problem are increased as it suffers from the CFL condition. The CFL condition requires that the time step should not be taken bigger than some computable quantity which depends on the discretization.

Rather than abandoning the steepest descent, the gradient is reconsidered in a different Sobolev space. We consider the Sobolev space $H_{2}^{2}$ which is $R^{N}$ with the inner product

$$
\begin{aligned}
\langle x, y\rangle_{s}= & \left\langle D_{00}(x), D_{00}(y)\right\rangle+\left\langle D_{11}(x), D_{11}(y)\right\rangle \\
& +\left\langle D_{22}(x), D_{22}(y)\right\rangle .
\end{aligned}
$$

It can be shown that the space defined by (32) is complete. The desired Sobolev gradient $\nabla_{s} F(u)$ in $H_{2}^{2}$ is found by solving

$$
\phi\left(D_{00}^{t} D_{00}+D_{11}^{t} D_{11}+D_{22}^{t} D_{22}\right) \phi \nabla_{s} F(u)=\phi \nabla F(u) .
$$

\section{Test Problems and Numerical Results}

Numerical experiments for the solution of nonlinear KGE were performed as follows. A system of $N$ nodes was set up with $\delta_{x}$ as internodal spacing. The function $u$ was then evolved. The programs were terminated when the infinity norm of the residual function $M(u)$ was less than $10^{-6}$.

Example 4. In this example we consider the nonlinear KGE with cubic nonlinearity

$$
\begin{aligned}
\frac{\partial^{2} u}{\partial t^{2}}+\alpha \frac{\partial^{2} u}{\partial x^{2}}+\beta u+\gamma u^{3}= & f(x, t), \\
& x \in \Omega=[0,1], 0<t \leq T
\end{aligned}
$$

with constants $\alpha=-1, \beta=0$, and $\gamma=1$ and the initial conditions

$$
\begin{gathered}
u(x, 0)=B \tan (K x), \quad 0 \leq x \leq 1 \\
u_{t}(x, 0)=B c K \sec ^{2}(K x), \quad 0 \leq x \leq 1
\end{gathered}
$$

The analytical solution to this problem is

$$
u(x, t)=B \tan (K(x+c t))
$$

where $B=\sqrt{\beta / \gamma}$ and $K=\sqrt{-\beta / 2\left(\alpha+c^{2}\right)}$.

Example 4 is solved by using both Euclidean and Sobolev gradients. We set $\delta_{t}=0.2$ for the time increment. For five time steps, results are summarized in Table 1 . Here $\lambda$ is the maximum step size that could be used to obtain convergence with both $L_{2}$ and $H_{2}^{2}$ gradient. Iterations denote the total number of minimization steps to reach convergence. CPUs denotes the time in seconds taken to reach convergence.

From Table 1, it can be seen that as we increase the number of nodes the $L_{2}$ gradient is going to be inefficient, but this is not in the case of Sobolev gradient.

The graphs of analytical solution and numerical solution obtained by the Sobolev gradient method for $t=4$ are shown in Figures 1 and 2. Figures 3 and 4 show the surface plot of the obtained solution.

In Tables 2 and 3, a comparison is shown between the Sobolev gradient method and LBM [20]. The $L_{2}, L_{\infty}$, 
TABLE 3: Comparison of $L_{\infty}, L_{2}$, and RMS errors for Example 4 at $c=0.05$ between Sobolev gradient method and LBM [20].

\begin{tabular}{ccccccccccc}
\hline \multirow{9}{*}{$t$} & \multicolumn{9}{c}{ Sobolev descent } & \multicolumn{3}{c}{ LBM } \\
& Nodes & $\delta_{t}$ & $L_{\infty}$ & $L_{2}$ & RMS & Nodes & $\delta_{t}$ & $L_{\infty}$ & $L_{2}$ \\
\hline 1 & 20 & 0.1 & $5.7169 \times 10^{-6}$ & $1.3781 \times 10^{-5}$ & $3.5582 \times 10^{-6}$ & 100 & $5.0 \times 10^{-5}$ & $5.6970 \times 10^{-5}$ & $2.9718 \times 10^{-4}$ & $2.9570 \times 10^{-5}$ \\
2 & 15 & 0.5 & $3.5225 \times 10^{-6}$ & $8.3370 \times 10^{-6}$ & $2.1526 \times 10^{-6}$ & 100 & $5.0 \times 10^{-5}$ & $7.4878 \times 10^{-5}$ & $3.8699 \times 10^{-4}$ & $3.8507 \times 10^{-5}$ \\
3 & 15 & 0.5 & $6.2838 \times 10^{-6}$ & $1.3009 \times 10^{-5}$ & $3.3589 \times 10^{-6}$ & 100 & $5.0 \times 10^{-5}$ & $1.1972 \times 10^{-4}$ & $5.2203 \times 10^{-4}$ & $5.1944 \times 10^{-5}$ \\
4 & 30 & 0.5 & $1.2084 \times 10^{-6}$ & $3.4327 \times 10^{-5}$ & $6.2672 \times 10^{-7}$ & 100 & $5.0 \times 10^{-5}$ & $1.4008 \times 10^{-3}$ & $4.4143 \times 10^{-4}$ & $4.3924 \times 10^{-5}$ \\
\hline
\end{tabular}

TABLE 4: Comparison of RMS errors for Example 4 between Sobolev descent, SMLP [21], and RBF [11].

\begin{tabular}{lcccc}
\hline$t$ & $E(u)$ & \multicolumn{3}{c}{ RMS } \\
& & SMLP & RBF & Sobolev descent \\
\hline 1 & 0.0585 & $5.5509 \times 10^{-8}$ & $1.7772 \times 10^{-7}$ & $3.5582 \times 10^{-6}$ \\
2 & 0.0686 & $1.1903 \times 10^{-7}$ & $1.5306 \times 10^{-7}$ & $2.1526 \times 10^{-6}$ \\
3 & 0.0795 & $1.3990 \times 10^{-7}$ & $1.7190 \times 10^{-7}$ & $3.3589 \times 10^{-6}$ \\
4 & 0.0815 & $1.0784 \times 10^{-7}$ & $1.9997 \times 10^{-7}$ & $6.2672 \times 10^{-7}$ \\
\hline
\end{tabular}

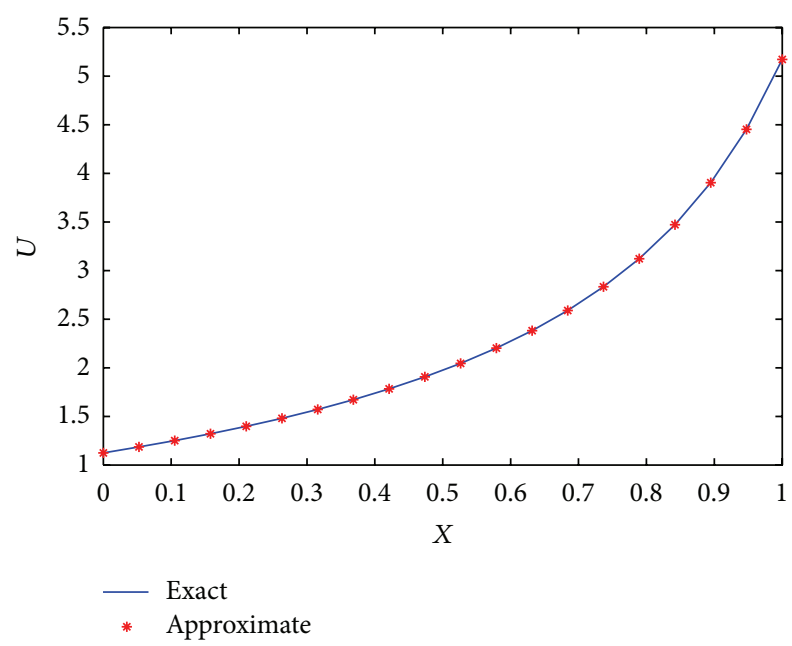

FIGURE 1: Numerical and exact solution of Example 4 at $t=4$ with $\delta_{t}=0.1, N=20$, and $c=0.5$.

and Root-Mean-Square (RMS) errors are obtained for $t=$ $1,2,3,4$.

Results in Tables 2 and 3 show the better accuracy of the proposed method than from LBM.

In Table 4 RMS value obtained by the Sobolev gradient method is compared with RBF and SMLP methods given in $[11,21]$. The values of energy $E(u)$ obtained by the proposed method for different time levels are also given.

Table 4 shows that the results obtained by the proposed method in terms of accuracy are comparable with other numerical schemes. The energy $E(u)$ remains invariant at different time levels.

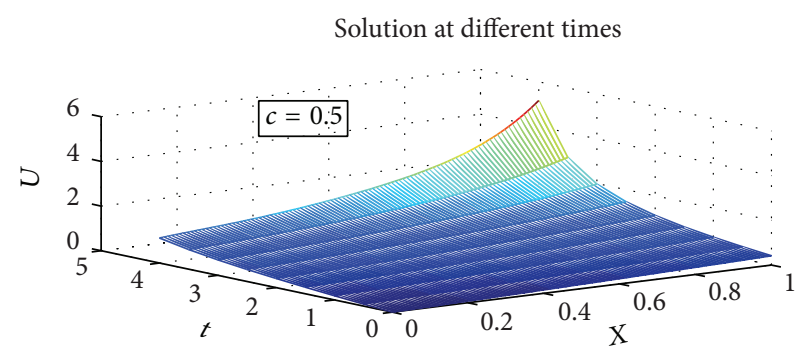

FIGURE 2: Space-time graph of Example 4 up to $t=4$ with $\delta_{t}=0.1$, $N=20$, and $c=0.5$.

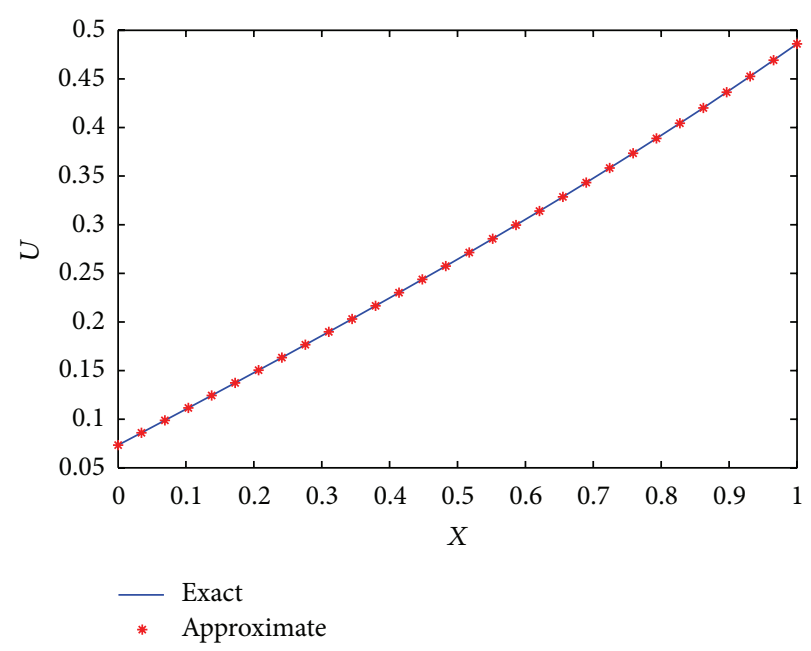

FIgURE 3: Numerical and exact solution of Example 4 at $t=4$ with $\delta_{t}=0.5, N=30$, and $c=0.05$.

Example 5. Consider the nonlinear KGE with quadratic nonlinearity

$$
\begin{aligned}
\frac{\partial^{2} u}{\partial t^{2}}+\alpha \frac{\partial^{2} u}{\partial x^{2}}+\beta u+\gamma u^{2}= & f(x, t), \\
& x \in \Omega=[0,1], 0<t \leq T
\end{aligned}
$$

with constants $\alpha=-1, \beta=0$, and $\gamma=1$ and initial conditions

$$
\begin{array}{cc}
u(x, 0)=0, & 0 \leq x \leq 1, \\
u_{t}(x, 0)=0, & 0 \leq x \leq 1 .
\end{array}
$$

The right-hand side function is

$$
f(x, t)=6 x t\left(x^{2}-t^{2}\right)+x^{6} t^{6}
$$


TABLE 5: Comparison of $L_{\mathrm{o}}, L_{2}$, and RMS errors for Example 5 at $c=0.05$ between Sobolev gradient method and LBM [20].

\begin{tabular}{|c|c|c|c|c|c|c|c|c|c|c|}
\hline \multirow{2}{*}{$t$} & \multicolumn{5}{|c|}{ Sobolev descent } & \multicolumn{5}{|c|}{ LBM } \\
\hline & Nodes & $\delta_{t}$ & $L_{\infty}$ & $L_{2}$ & RMS & Nodes & $\delta_{t}$ & $L_{\infty}$ & $L_{2}$ & RMS \\
\hline 1 & 10 & 0.1 & $2.4302 \times 10^{-2}$ & $5.3120 \times 10^{-2}$ & $1.6890 \times 10^{-2}$ & 100 & $5.0 \times 10^{-5}$ & $5.8742 \times 10^{-4}$ & $1.9270 \times 10^{-3}$ & $1.9174 \times 10^{-4}$ \\
\hline 3 & 10 & 0.1 & $8.6234 \times 10^{-3}$ & $1.9334 \times 10^{-2}$ & $6.1398 \times 10^{-3}$ & 100 & $5.0 \times 10^{-5}$ & $1.5139 \times 10^{-2}$ & $4.9465 \times 10^{-2}$ & $4.9219 \times 10^{-3}$ \\
\hline 5 & 10 & 0.1 & $4.3457 \times 10^{-3}$ & $5.0021 \times 10^{-3}$ & $1.6089 \times 10^{-3}$ & 100 & $5.0 \times 10^{-5}$ & $6.3219 \times 10^{-2}$ & $9.3035 \times 10^{-2}$ & $1.2970 \times 10^{-2}$ \\
\hline 7 & 10 & 0.1 & $1.1123 \times 10^{-3}$ & $2.1457 \times 10^{-3}$ & $6.7938 \times 10^{-4}$ & - & - & - & - & - \\
\hline
\end{tabular}

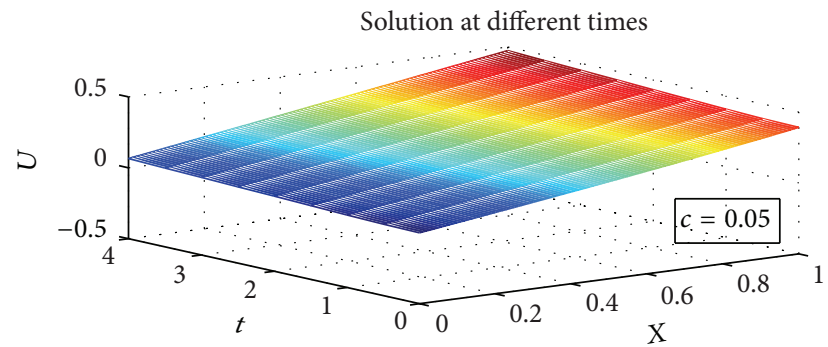

FIgURE 4: Space-time graph of Example 4 up to $t=4$ with $\delta_{t}=0.5$, $N=30$, and $c=0.05$.

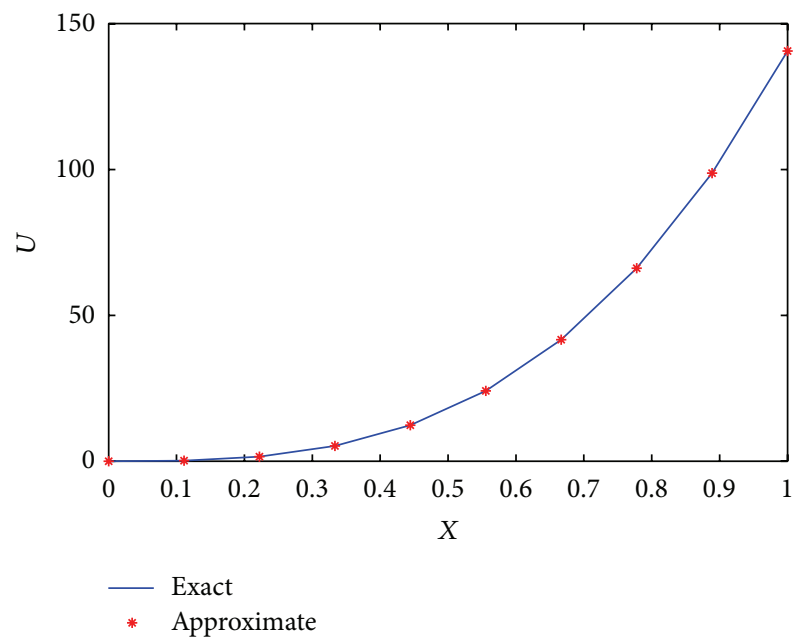

Figure 5: Numerical solution of Example 5 at $t=5$ with $\delta_{t}=0.1$, $N=10$, and $c=0.05$.

and the analytical solution to this problem is

$$
u(x, t)=x^{3} t^{3}
$$

We get the boundary function $g(x, t)$ from the exact solution.

The graph of the approximated and exact solutions at $t=$ 4 is shown in Figure 5 and the space-time graph using Sobolev gradient method is shown in Figure 6.

Table 5 shows the comparison between the Sobolev gradient method and LBM [20]. The $L_{2}, L_{\infty}$, and Root-MeanSquare (RMS) errors are also calculated for $t=1,2,3,4,5$.

Once again the results show the better accuracy of the proposed method than LBM.

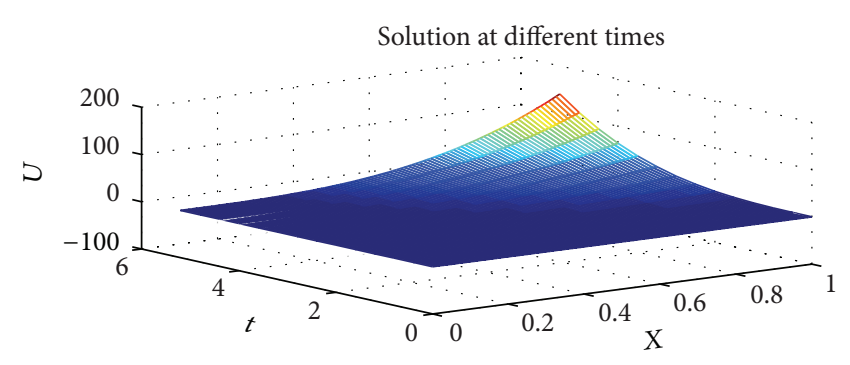

FIGURE 6: Space-time graph of Example 5 up to $t=5$ with $\delta_{t}=0.1$, $N=10$, and $c=0.05$.

\section{Summary and Conclusions}

In this paper, we proposed a numerical scheme to solve the nonlinear KGE using Sobolev gradients. Table 1 shows that standard steepest descent using the $L_{2}$ gradient compels us to choose a very small step size $\lambda$, leading to a huge number of iterations and sometimes a failure to reach convergence.

Steepest descent defined in an appropriate Sobolev space, on the other hand, uses a very large $\lambda$ and fewer iterations. So the Sobolev gradient technique is preferable to the usual steepest descent technique as the spacing of the grid is made finer.

Sobolev gradient techniques may offer definite benefits in certain cases; for example, the choice of initial guess does not affect convergence of the method. The results of two examples are compared with the LBM in [20]. Our scheme uses a large time step $\delta_{t}$ as compared with the LBM and gives better numerical results in terms of accuracy. In these two examples results show that, for larger values of time $t$, the Sobolev gradient approach is better than the LBM. The choice of optimal metric defined in Sobolev space can further improve the numerical results, but how to choose such a metric is still an important question to be investigated.

\section{Conflict of Interests}

The authors declare that there is no conflict of interests regarding the publication of this paper.

\section{Acknowledgment}

The first author is thankful to University of Punjab for providing research grant via D/605/Est. 1 dated 24-02-2015. 


\section{References}

[1] A.-M. Wazwaz, "New travelling wave solutions to the Boussinesq and the Klein-Gordon equations," Communications in Nonlinear Science and Numerical Simulation, vol. 13, no. 5, pp. 889-901, 2008.

[2] B. Bulbul, M. Sezer, and W. Greiner, Relativistic Quantum Mechanics-Wave Equations, Springer, Berlin, Germany, 3rd edition, 2000.

[3] P. J. Caudrey, J. C. Eilbeck, and J. D. Gibbon, "The sine-Gordon equation as a model classical field theory," Il Nuovo Cimento B Series, vol. 25, no. 2, pp. 497-512, 1975.

[4] A. Biswas, A. Yildirim, T. Hayat, O. M. Aldossary, and R. Sassaman, "Soliton perturbation theory for the generalized Klein-Gordon equation with full nonlinearity," Proceedings of the Romanian Academy A, vol. 13, no. 1, pp. 32-41, 2012.

[5] A. Biswas, C. Zony, and E. Zerrad, "Soliton perturbation theory for the quadratic nonlinear Klein-Gordon equation," Applied Mathematics and Computation, vol. 203, no. 1, pp. 153-156, 2008.

[6] A. Biswas, G. Ebadi, M. Fessak et al., "Solutions of the perturbed Klein-Gordon equations," Iranian Journal of Science and Technology A, vol. 36, no. 4, pp. 431-452, 2012.

[7] Sirendaoreji, "Auxillary equation method and new solutions of Klein-Gordon equations," Chaos, Solitons \& Fractals, vol. 31, no. 4, pp. 943-950, 2007.

[8] Sirendaoreji, "A new auxiliary equation and exact travelling wave solutions of nonlinear equations," Physics Letters A, vol. 356, no. 2, pp. 124-130, 2006.

[9] X. Li and B. Y. Guo, "A legendre spectral method for solving nonlinear Klein-Gordon equation," Journal of Computational Mathematics, vol. 15, no. 2, pp. 105-126, 1997.

[10] B. Y. Guo, X. Li, and L. Vazquez, "A Legendre spectral method for solving the nonlinear Klein-Gordon equation," Mathematics Applied and Computation, vol. 15, no. 1, pp. 19-36, 1996.

[11] M. Dehghan and A. Shokri, "Numerical solution of the nonlinear Klein-Gordon equation using radial basis functions," Journal of Computational and Applied Mathematics, vol. 230, no. 2, pp. 400-410, 2009.

[12] M. A. M. Lynch, "Large amplitude instability in finite difference approximations to the Klein-Gordon equation," Applied Numerical Mathematics, vol. 31, no. 2, pp. 173-182, 1999.

[13] J. W. Neuberger, Sobolev Gradients and Differential Equations, vol. 1670 of Lecture Notes in Mathematics, Springer, New York, NY, USA, 1997.

[14] S. Sial, J. Neuberger, T. Lookman, and A. Saxena, "Energy minimization using Sobolev gradients: application to phase separation and ordering," Journal of Computational Physics, vol. 189, no. 1, pp. 88-97, 2003.

[15] J. Karatson and L. Loczi, "Sobolev gradient preconditioning for the electrostatic potential equation," Computers \& Mathematics with Applications, vol. 50, no. 7, pp. 1093-1104, 2005.

[16] J. Karatson and I. Farago, "Preconditioning operators and Sobolev gradients for nonlinear elliptic problems," Computers \& Mathematics with Applications, vol. 50, no. 7, pp. 1077-1092, 2005.

[17] B. M. Brown, M. Jais, and I. W. Knowles, "A variational approach to an elastic inverse problem," Inverse Problems, vol. 21, no. 6, pp. 1953-1973, 2005.

[18] I. Knowles and A. Yan, "On the recovery of transport parameters in ground-water modelling," Journal of Computational and Applied Mathematics, vol. 171, no. 1-2, pp. 277-290, 2004.
[19] J. Garcia-Ripoll, V. Konotop, B. Malomed, and V. Perez-Garcia, "A quasi-local Gross-Pitaevskii equation for attractive BoseEinstein condensates," Mathematics and Computers in Simulation, vol. 62, no. 1-2, pp. 21-30, 2003.

[20] Q. Li, Z. Ji, Z. Zheng, and H. Liu, "Numerical solution of nonlinear Klein-Gordon equation using lattice Boltzmann method," Applied Mathematics, vol. 2, no. 12, pp. 1479-1485, 2011.

[21] F. Yin, T. Tian, J. Song, and M. Zhu, "Spectral methods using legendre wavelets for nonlinear Klein-Gordon/Sine-Gordon equations," Journal of Computational and Applied Mathematics, vol. 275, pp. 321-334, 2015. 


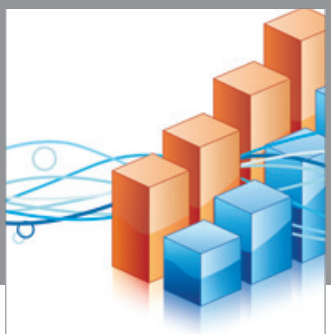

Advances in

Operations Research

vatem alat4

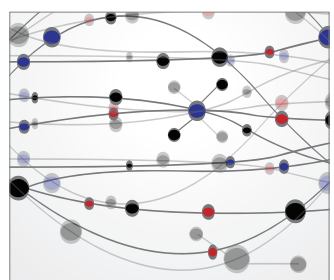

\section{The Scientific} World Journal
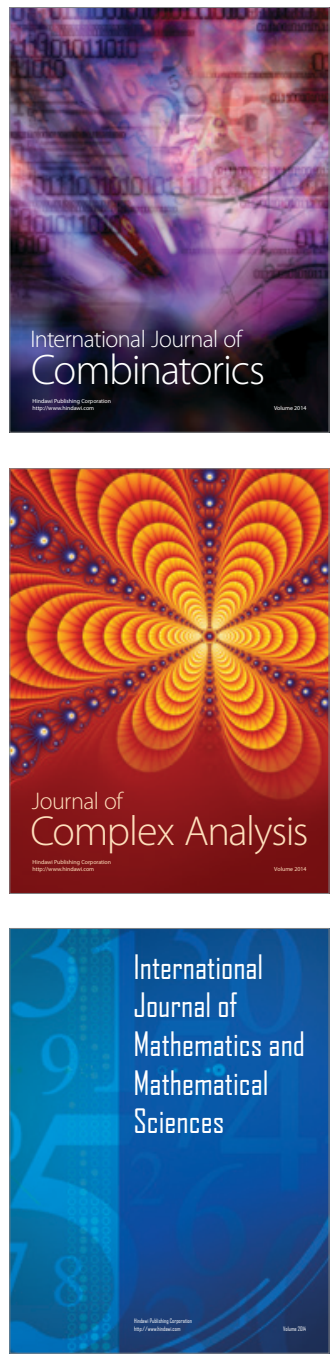
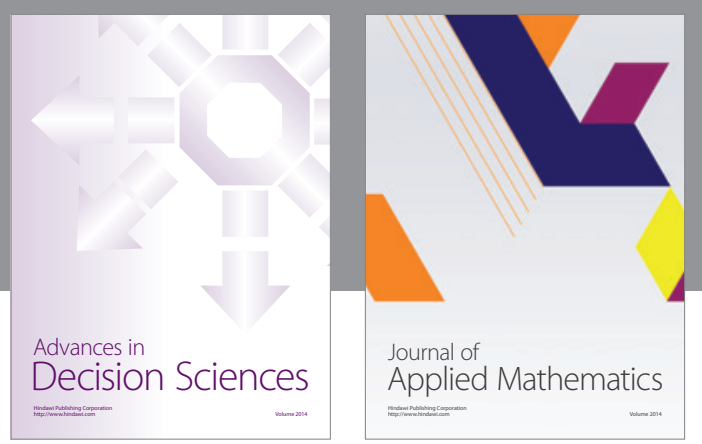

Algebra

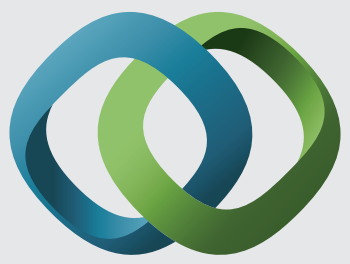

\section{Hindawi}

Submit your manuscripts at

http://www.hindawi.com
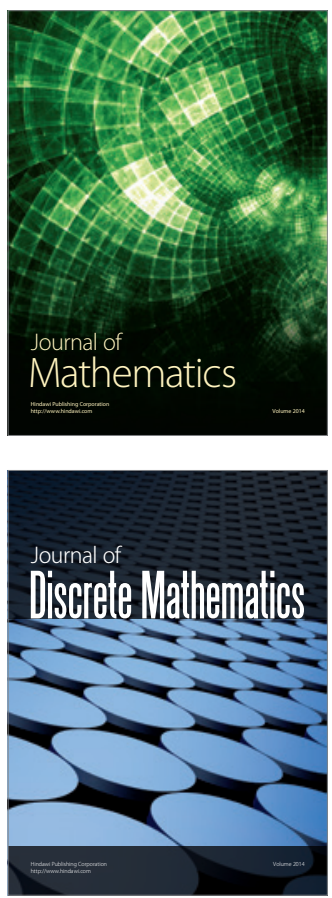

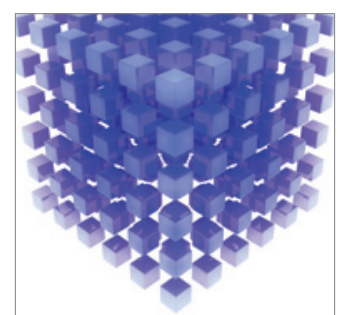

Mathematical Problems in Engineering
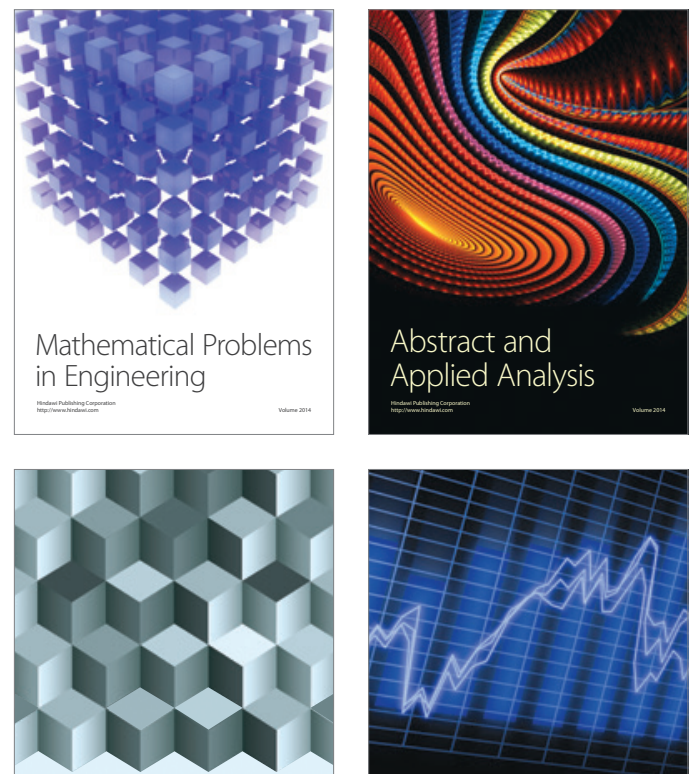

Journal of

Function Spaces

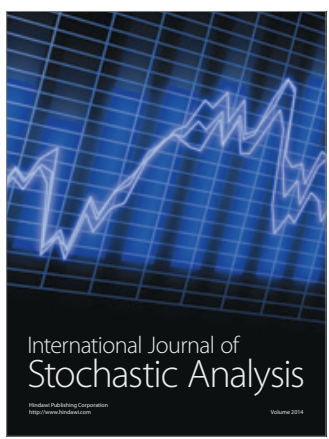

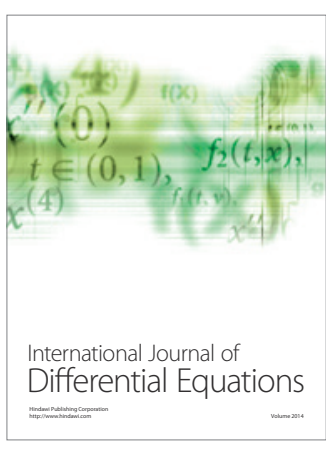
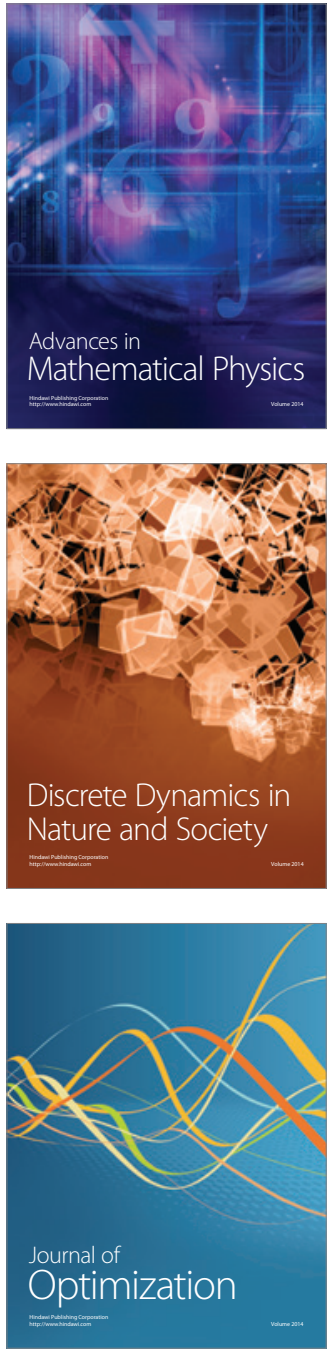